\title{
Modulation of a Laser Diode with a Transistor field effect metal-oxide-semiconductor
}

\author{
Diego Sabino ${ }^{1}$, Jaime H. Puebla ${ }^{2}$, Roberto Vázquez ${ }^{3}$ \\ ${ }^{I}$ M.E Student, Systems Engineering Department ESCOM-IPN, D.F. México. \\ ${ }^{2}$ Professor and Head, Systems Engineering Department, ESCOM-IPN, D.F. México. \\ ${ }^{3}$ Assistant Professor, Systems Engineering Department, ESCOM-IPN, D.F. México.
}

\begin{abstract}
For industrial applications it is necessary that the test systems are portable, for example measuring the concentration of pollutants in water or characterization of processes that occur in plants or other organisms be developed in situ and in vivo. The need to make the system portable, leads us to develop a driver that automatically controls the intensity of power and modulation of the laser, measuring the temperature of the respective material to be analyzed. Presents the implementation of the characteristics of a field effect transistor metal-oxide-semiconductor as a tool for modulating the laser diode. The result is a driver and cost effective that can be used in various photothermal phenomena.
\end{abstract}

Keywords: Laser, MOSFET, Photothermal techniques

\section{INTRODUCTION}

Photothermal techniques have demonstrated their potential for measuring thermal properties of materials. In particular technique fotopiroeléctrica (FPE) offers the advantage of facilitating the realization of measurements at different temperatures in a relatively easy and inexpensive.

Due to the good thermal contact takes place between the pyroelectric sensor and sample liquid or semiliquid, the FPE technique has become a very useful tool for the detection of phase transitions in these systems and for the study of phenomena that can take place near so-called critical points. This opens many expectations for use on an industrial scale, so that the proposed experimental systems compact, inexpensive, and portable is impetuous.

Regarding the modulated light source to excite the signal FT is scanned using laser diodes (LD) and light emitting diodes (LED) of moderate power available at low cost in a wide spectral region and which can be modulated in a wide range of frequency. It is increasingly common for its practicality, the use of photoacoustic techniques for identification of specific substances.

The use of these techniques, involves among other things the modulation of a radiation source which exposes the sample to obtain its characterization [1]. In most cases it is choose powerful lamps that can be modulated with external devices. All of this gives robustness to the experiment, besides it became more expensive [2].

Find modulated radiation sources that involve a lower cost and also remain practical is the objective of this work. A diode laser with a suitable modulation can be a viable substitute for this kind of sources. The diode laser it's going to use a TTL signal, which will be generated by a medium scale microcontroller the PIC16F876.

The uses of a driver will aloud the correct modulation of the Laser Diode to obtain the desired modulation results in a wide frequency range through its feed current. It is important to note that this project is part of another system which will be in charge of collecting information through a photoacoustic chamber, at which will be incorporate a microphone for data collection.

Later this information will be amplified by a lock-in amplifier, which function is to amplify the very low amplitude signals. Finally, through a Graphical User Interface (GUI) will be displayed in screen the graphs of the results obtained.

\section{DEVELOPMENT}

To implement a useful radiation source it's necessary to take various considerations. One of them is the type of sample that is looking to characterize, for this factor determines, among other things, the necessary potency of the power source to generate the optimal photoacoustic signals [3]. Also relevant is determinate the configuration in this experiment, in some cases, the space is limited, and it seek an arrangement with small 
dimensions. For this work, where considered biological type samples and a small space for the development of the experiment.

For the stage of control and conditioning in our system. The circuit show in Figure 1. Shows the schematic of the control stage to take care of handling the Field Effect Transistors, it is important to mention that the light emitting diodes are polarize to different voltages, because of this it's necessary to use voltage regulators as the case of the diode light emitting to will be polarize.

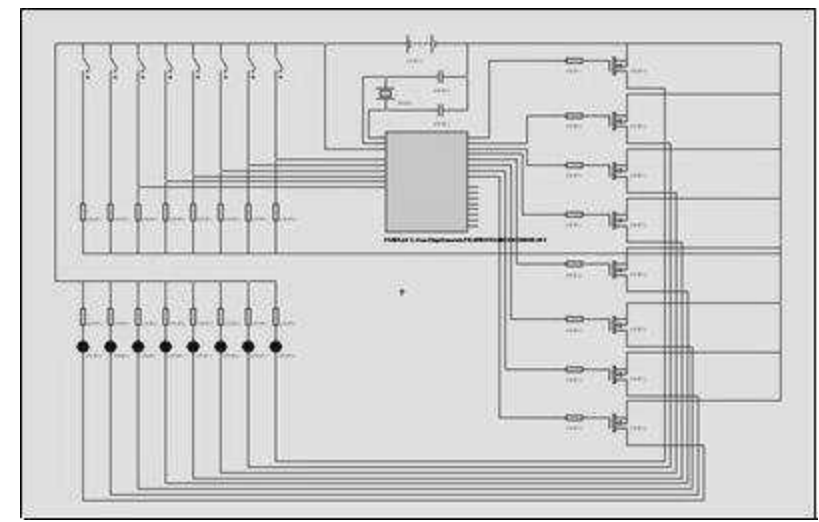

Figure 1. Schematic of the Control Stage.

\section{CHARACTERISTICS OF THE LASER DIODE USED}

One of the advantages of laser diodes is their versatility. It can easily find diodes with different wavelengths and power, depending on the application that seeks to give [4].

For this case it is necessary to irradiate biological samples, which means that to get a proper insight into these, the use of a diode laser with a wavelength between $600 \mathrm{~nm}$ east-1200nm can perform this task [5]. Regarding the potency diode relates, whereas the samples are small, the potency should not be higher than 10 $\mathrm{mW}$.

In our project we used infrared AlGaAs laser diodes EGISMOS brand with a wavelength of 780nm and a potency output of $5 \mathrm{~mW}$, with key D6mm costing no more than 30 dollars. The justification for using these Laser Diodes was because is a semiconductor laser and additionally contains substances that are not insulating possess conductivity lower to metals. Today the most used diode is that of gallium arsenide (GaAs), which may exist in the market with different wavelengths. GaAs conl $=780,830$ and $904 \mathrm{~nm}$ (infrared lasers). GaAs conl = 630, 633, 650 and $670 \mathrm{~nm}$ (red laser).

The active medium is a p-n junction in which by passing a current greater than a given threshold occurs population inversion through recombination of charge carriers at the junction releasing energy as photons, which are reflected in the polished faces to the junction again and it provokes the retro-causing optical power necessary to sustain the emission. It can operate in $\mathrm{CW}$ (continuous way or continuous duty) with efficiency in excess of $50 \%$.

These lasers are more efficient, cheaper and smaller, and have remained leaders in many scientific and technological applications. For example one of them with a wavelength of $780 \mathrm{~nm}$ and a potency of $5 \mathrm{~mW}$, with the key D6mm costing no more than 30 dollars.

In total eight Laser Diodes were used at different wavelengths, also with different bias. Among the specifications of each laser diode is detailed electrical characteristics of each of them. The following describes the electrical properties more important for our case which is pulse width modulation of the laser diode.

Figure 2 shows the variation of the potency emitted by the laser diode EGISMO that is used which was employed in this work, based on the applied current. In it can be observed two regions: in the first one the behavior is linear and correspond to low currents where the LED acts as a common, the second region corresponds to the currents in which the diode behaves as a laser but still retains the linear behavior.

This behavior is typical of laser diode when operating in free mode, without any feedback. 


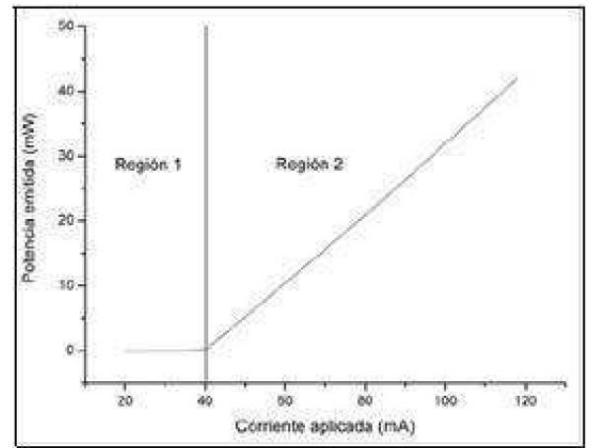

Figure 2.Diagram of Potency emitted (P) vs. Applicant current (I) laser diode EGISMO use in this work.

The two regions are separated by a zone of inflection, this indicates that the current has become a value large enough to achieve the population inversion and start the laser emission. Since the observed behavior is linear in these regions is possible to find the value of current setting where lines intersect, this value is known as the threshold current and this value correspond to the current emission in which the laser is stabilized.

Figure 3. They have a wavelength and potency that are the parameters that define the most important characteristics of this type of laser diode used in our case. It should be mentioned that the graph describe in Figure 3 is a representation of one wavelength vs. Laser Diode Potency. In the first of these the stimulated emission occurs in a relatively wide region of the semiconductor, which in the region of gain and in the resonating longitudinal which are permitted within the semiconductor.

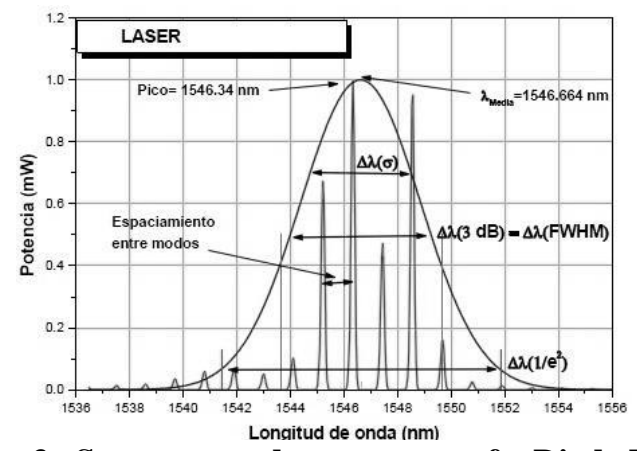

Figure 3. Spectrum and parameters of a Diode Laser.

Importantly, Great care was taken with each of them to polarize because these laser diodes are sensitive to voltage variations. Also take special care to not drop below the threshold current and to keep the area in which the modulation efficiency is linear with frequency. In some devices can reach the GHz región. The drawbacks of these possibilities, it should be noted that the spectrum instability occurs in relation to temperature and power.

\section{SOURCE MODULATION}

Process which this paper is focus. The modulation of the laser diode is required to obtain a different exposure in each of the experiments, involving different results. The sample exposure to radiation source ranging from 10 to hundreds of nanoseconds. This exposure is controlled by a pulse TTL from 4.5v to 5.v. To control exposure are used transistors, based on the electric field to control a channel conductivity of a material semiconductor. However, an additional characteristic of this transistor type Metal / Oxide / Semiconductor is that the gate terminal $(\mathrm{G})$ is constructed from this material. The oxide is insulate, so the gate current is negligible, far less than the transistors in common use. 


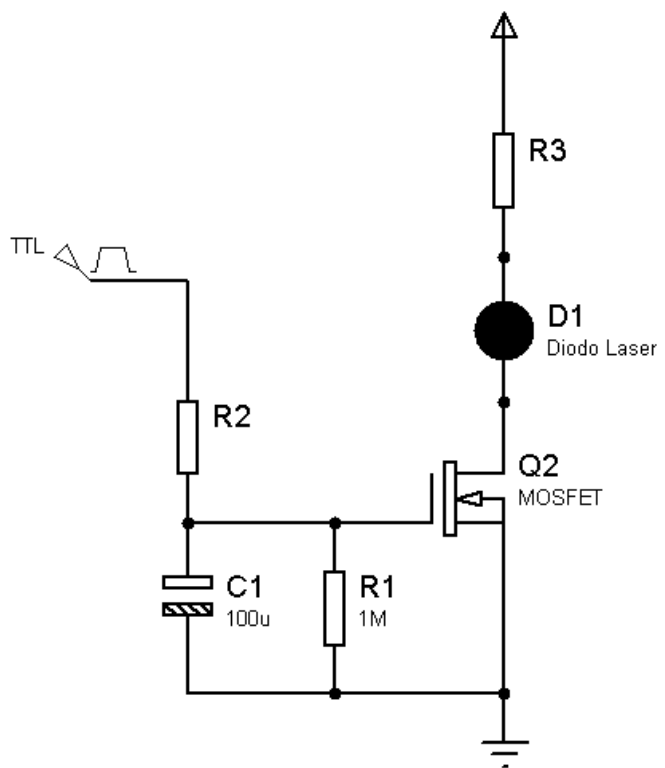

Figure 4. Driver Diagram.

Figure 4 shows the connection diagram of the components required for modulating the laser diode using the properties of a MOSFET (Q2).

The resistors R2 and R3 vary depending on the power required by the laser diode. While $\mathrm{C} 1$ and $\mathrm{R} 1$ are connected as a low pass filter, preventing the generation of noise even when the TTL input signal is working at high frequencies.

\section{RESULTS}

Figure 5 shows the voltage signals entering the circuit as a TTL signal (1) and also the power supplied to the laser diode.

(2).

(1)

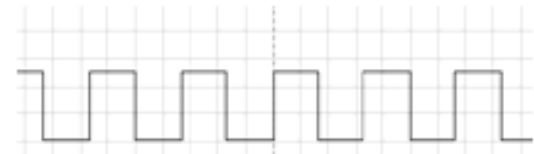

(2)

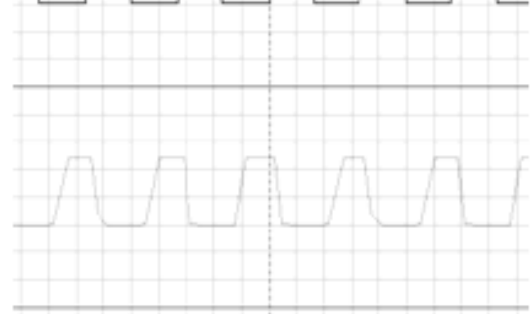

Figure 5. Circuit behavior.

One can see that although there is a phase shift in the laser diode power supply, with respect to the TTL input signal, the circuit behaves as a stable modulator. Note that although this experiment the laser diode power supply is $5 \mathrm{~V}$ as TTL signal. The same circuit can be used with higher supply voltages. In tests conducted with the laser diode have the following figures. Figure 6 shows the dimensions of the laser diodes that employ in the project and the beam intensity emitted by the laser diode. 


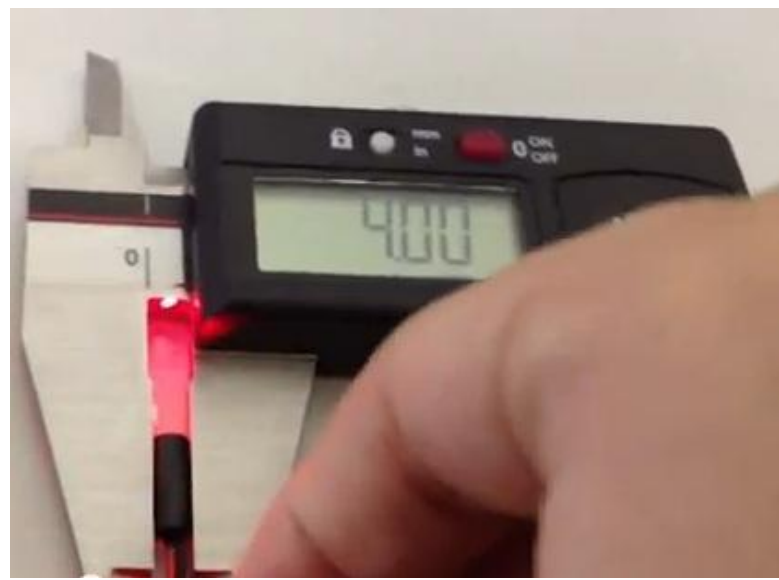

Figure 6. Laser Diode Features employee.

Figure 7 shows the computer that was used to modulate pulse width in Laser Diode.

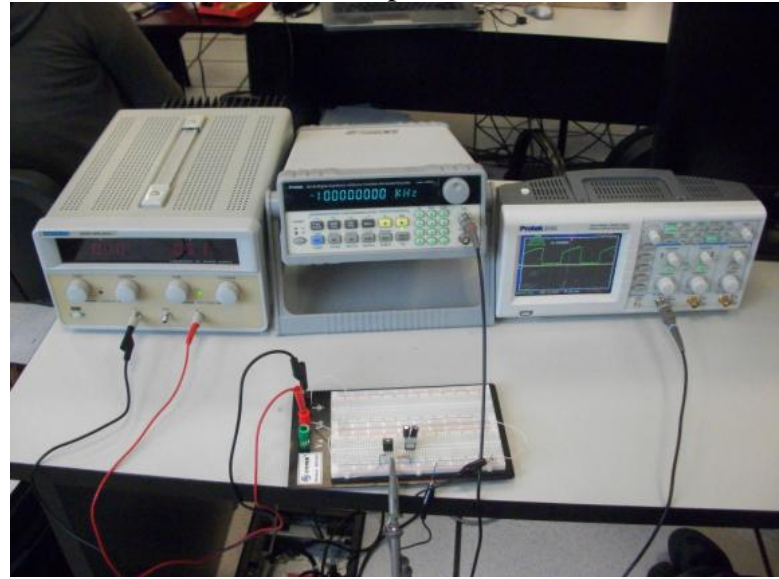

Figure 7. Equipment used for testing.

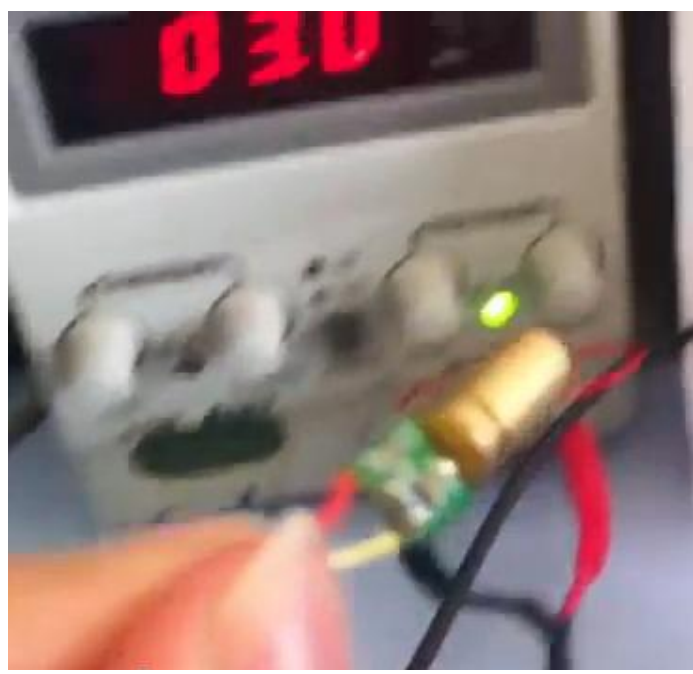

Figure 8. Laser manipulation in the Amplitude Modulation.

It is important to remember that since this part is only the first stage of a multidisciplinary project, there remains the photoacoustic chamber, which is where you will find the sample to obtain results of the photothermal technique is developing here. 


\section{CONCLUSIONS}

The implementation of a MOSFET device for modulating a laser diode can be replaced with complex arrangements of gas lamps or lasers coupled to other devices, for sample radiation photoacoustic techniques. Reducing the cost and space required for these experiments.

\section{REFERENCES}

1). Akiyuki Minamade; Yoshiaki Tokunaga Material Evaluation with New Modulation Method in Photoacoustic Technique. 10.1109/ULTSYM.2009.0632.

2). Zoltán Bozóki; Miklós Szakáll; Árpad Mohácsi, Gábor Szabó; Zsolt Bor Diode Laser Based Photoacoustic Humidity Sensors. Sensors and Actuators B 91 219-226 (2003)

3). V. Horká; S. Civis, Li-Hong Xu; R.M. Lees Laser Diode Photoaouctic detection in the infrared and near infrared spectral Ranges. The analyst PAPER DOI: 10.1039/b503838c 2005.

4). Thomas J. Allen; Paul C. Beard Dual wavelength laser diode excitation source for 2D photoacoustic imaging. Biomedical Thermoacoustics Proc. Of SPIE Vol. 6437 64371U-1.

5). Thomas J. Allen; Paul C. Beard Pulsed near-infrared Laser Diode Excitation System for Biomedical Photoacoustic Imaging. Optics Letters. Vol.31,No. 23. 2006.

6). Kindereit, U.; Woods, G.; Jing Tian; Kerst, U.; Leihkauf, R.; Boit, C. Quantitative Investigation of Laser Beam Modulation in Electrically Active Devices as Used in Laser VoltageProbing.Volume:710.1109/TDMR.2007.898074.

7). Dharamsi, A.N.; Lu, Y.; Bullock, A.M. Effects of amplitude and frequency modulation with diode lasers, using higher harmonic detection Volume: 2 10.1109/LEOS.1995.484739.

8). Takamiya, S.; Kitasawa, F.; Nishizawa, J.-I. Amplitude modulation of diode laser light in millimeterwave region. Volume: 56. 10.1109/PROC.1968.6202.

9). Streifer, W.; Yevick, D.; Paoli, T.; Burnham, R. Analysis of cleaved coupled-cavity (C3) diode lasers - Part II: Frequency modulation, above threshold operation, and residual amplitude modulation.Volume:21.10.1109/JQE.1985.107279.

10). Arce-Diego, J.L.; Giuliani, G.; Cepeda-Echevarria, R.M.; Pereda-Cubian, D.; Fernandez-Fernandez, L.A. Effects of undesired external reflections on a laser diode feedback interferometer for measurement of displacements without ambiguity. Volume:2.10.1109/LEOS.2001.968983 